\title{
Construction of entrepreneurship evaluation system for college graduates -Empirical analysis based on BP neural network
}

\author{
Xiaoyi Wang ${ }^{1}$ and Hui Che ${ }^{1}$ \\ ${ }^{1}$ School of safety engineering, Shenyang Aerospace University Shenyang China
}

\begin{abstract}
In order to accurately and efficiently evaluate the entrepreneurial success rate and the risks in the entrepreneurial process of college graduates. BP Neural Network is used to establish the evaluation system of College Students' entrepreneurship process, making contributions to the underwriting system of entrepreneurship insurance. 12 influence factors are selected as input variables, and the neuron weight and learning rateare adjusted in the training process.
\end{abstract}

\section{Introduction}

In recent years, the problem of entrepreneurship has been perplexing society and universities after graduation. Therefore, many scholars studied the source and path of entrepreneurial opportunities for college students. But the most comprehensive index Bank of entrepreneurial opportunity is Timmons' entrepreneurship opportunity evaluation index, which includes 8 first-level indicators and 53 secondary indicators. But because of the many factors, the weight is difficult to measure, and it is difficult to use in the real venture opportunity evaluation. Pengyunfang ${ }^{[4]}$ puts forward the index system of comprehensive evaluation of entrepreneurship opportunity by using fuzzy comprehensive evaluation method under the framework of Timmons' entrepreneurial opportunity evaluation. But the fuzzy comprehensive evaluation method still has the problem of complex calculation and the determination of index weight has a strong subjectivity. Of course, Bhave maheshp ${ }^{[7]}$ established the process model of venture risk creation.

The above studies show that the factors that affect college students' Entrepreneurship present a complex nonlinear relationship. This paper studies how to promote the entrepreneurship and employment of college graduates.

\section{Research background}

Influenced by the COVID-19 epidemic and the downward pressure of the national economy, China's employment situation is particularly prominent.

\subsection{The number of college graduates is increasing}

This year, 10.71 million people have registered for the gaokao, and the number of college students is expected to reach a new high in the near future. In 2020, we will expand the enrollment scale of postgraduate students. Compared with the same year, this year's enrollment increased by nearly 190,000 .

\subsection{Solve the problems in the process of college students starting their own businesses}

College students often have the following difficulties in the process of starting a business.

1) College students lack social and business experience in starting a business, and it is difficult to grasp if they put themselves into the business society without guidance.

2) For college students who have just entered the society, their interpersonal skills are poor, and they don't know much about venue rental and equipment purchase.

\subsection{National policies promote innovation and entrepreneurship}

Promoting scientific and technological progress and innovation-driven development have become an objective requirement of China's economic and social development in the new era, and a strategic choice for realizing the great rejuvenation of the Chinese nation.

To sum up, the introduction of employment insurance can not only effectively alleviate the employment difficulties of college students, but also greatly promote the development of social economy. 


\section{Construction of BP neural network prediction model}

Based on the above analysis ideas, we obtain 12 variables that we want to input through the questionnaire, and establish the associated mathematical model.
In this algorithm, the input layer consists of twelve elements, in the whole operation process of the model, repeatedly before the signal propagation and error backward propagation and update its connection weights and threshold, the node gradually until the network output error reduced to an acceptable level or to set the number of learning, the final output results.

\subsection{Overview of BP neural network model}

We adopt a typical three-layer BP neural network model.

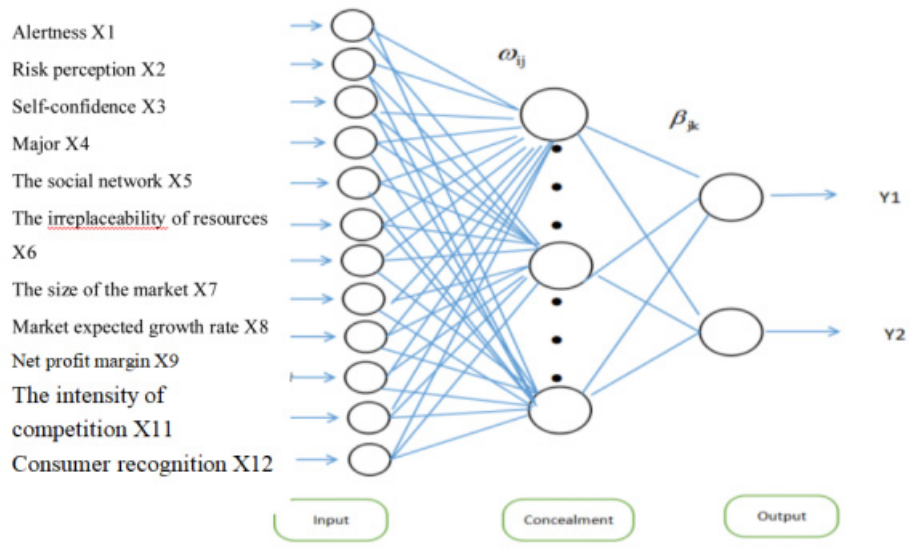

Figure 1 BP neural network structure diagram

\subsection{Selection of variables and reasons}

This paper summarizes three key influencing factors of entrepreneurial opportunities--- team, project and environment as primary indicators. Then the Timonns entrepreneurial evaluation indicators are summarized and classified, and 12 representative indicators are selected as the second-level indicators.

1) Entrepreneurial team. Entrepreneurs and their partners need to be able to identify and evaluate new markets in a timely manner and be able to develop them into new businesses. In addition to choosing vigilance, risk perception, confidence and major related to personal characteristics, this paper also adds irreplaceability of social network and resources as input variables according to research needs.

2) Entrepreneurial projects. When an entrepreneur finds an opportunity to start a business, he or she needs to transform the project into basic market economy and social value, which is the combined result of market size, expected market growth rate and net profit margin.

3) Entrepreneurial environment. It is the collection of a series of complex concepts. We should consider the market, economy, politics, society, etc.

Table 1: Input variables of entrepreneurial evaluation system for college graduates

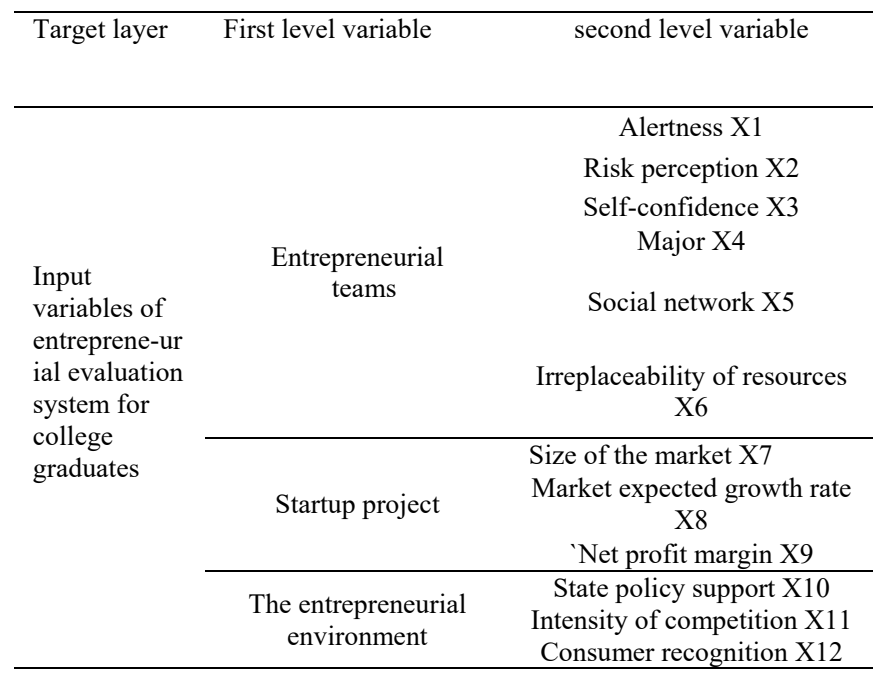




\subsection{Construction of BP neural network}

Step 1: Network initialization. $\mathrm{X}_{\mathrm{i}}$ represents the input of the ith neuron in the input layer,${ }^{i=1,2,3 \ldots . . .} ; \omega_{i j}$ is the weight value between the input layer $i$ and the hidden layer $\mathrm{j}, \mathrm{j}=1,2, \cdots, n ; f$ represents the activation function of the hidden layer; $\beta_{\mathrm{jk}}$ is the weight between the $\mathrm{j}$ neuron in the hidden layer jth and the kth neuron in the output layer. $\mathrm{Y}_{1}, Y_{2}$ represents the entrepreneurial success rate and the risk in the entrepreneurial process respectively. Sigmoid function is adopted as the activation function, and the formula is $f(x)=\frac{1}{1+\mathrm{e}^{-x}}$.

Step 2: Output vectors at each layer. Output layer output vector $b_{i}: b_{i}=f\left(h_{i}\right), i=1,2 \cdots 12 \quad h_{i}=\sum_{\mathrm{j}=1}^{n} f\left(\beta_{i j} c_{i}\right)$

The hidden layer output vector $c_{j}$ : $c_{j}=f\left(h_{j}\right), j=1,2 \cdots, n \quad h_{j}=\sum_{i=0}^{12} \omega_{i j} x_{i}$

Step 3: Error output. Let's say the expected value is a, the true value is $b$, the error is $E=\frac{1}{2}(a-d)^{2}$.

Therefore, the hidden layer error is: $\left.\mathrm{E}=\frac{1}{2} \sum r-f\left(h_{k}\right)\right]^{2}=\frac{1}{2} \sum_{k=1}^{l}\left[r-f\left(\sum_{j=0}^{\mathrm{n}} f\left(\beta_{i} c_{i}\right)\right)\right]$

The output layer error is: $\mathrm{E}=\frac{1}{2} \sum\left\{r_{j}-f\left[\sum_{j=0}^{m} \beta_{i j} f\left(\sum_{i=0}^{n} \omega_{i j} x_{i}\right)\right]\right\}$

Step 4: Minimize the error.

$$
\begin{aligned}
& \Delta \omega_{\mathrm{ij}}=-\frac{\partial E}{\partial \omega_{i j}}, i=0,1,2 \cdots, 12 ; j=0,1,2, \cdots, n \\
& \Delta \beta_{j k}=-\frac{\partial E}{\partial \beta_{j k}}, j=0,1,2 \cdots, n ; k=0,1,2, \cdots, l
\end{aligned}
$$

Step 5: Weight adjustment derivation process. Two error variables are set, Hidden layer: $\chi_{j}^{c}=-\frac{\partial E}{\partial h_{j}}$

Output layer: $\chi_{k}^{b}=-\frac{\partial E}{\partial h_{k}}$.Then we can calculate: $\Delta \omega_{\mathrm{ij}}=\mu \chi_{\mathrm{j}}^{\mathrm{c}} x_{i} \quad \Delta \beta_{\mathrm{jk}}=\mu \chi_{\mathrm{k}}^{b} y_{j}$

Combined with the above formula:

$$
\begin{aligned}
& \chi_{j}^{c}=-\frac{\partial E}{\partial h_{j}}=-\frac{\partial E}{\partial y_{j}} \bullet \frac{\partial y_{k}}{\partial h_{j}}=-\frac{\partial E}{\partial b_{j}} f^{\prime}\left(h_{j}\right) \\
& \chi_{\mathrm{k}}^{b}=-\frac{\partial E}{\partial h_{k}}=-\frac{\partial E}{\partial b_{k}} \bullet \frac{\partial b_{k}}{\partial h_{k}}=-\frac{\partial E}{\partial b_{k}} f^{\prime}\left(h_{k}\right)
\end{aligned}
$$

Therefore, the weight adjustment formula of the algorithm is obtained:

$$
\begin{aligned}
& \Delta \beta_{j k}=\mu \chi_{k}^{b} c_{j}=\mu\left(a_{k}-d_{k}\right) f^{\prime}\left(h_{k}\right) c_{j} \\
& \Delta \omega_{i j}=\mu \chi_{j}^{c} x_{i}=\mu\left(\sum_{i=1}^{12} \chi_{k}^{b} \beta_{j k}\right) f^{\prime}\left(h_{k}\right) x_{i} \beta_{j k}
\end{aligned}
$$

It can be seen that the determinants are: learning rate $\mu$, error variable $\chi$ and input variable (X or $\mathrm{Y}$ ).

\section{Verification and analysis of examples}

In order to verify the effectiveness of the model, we selected students who participated in large entrepreneurship projects and started businesses after graduation in some universities to fill in the questionnaire. A total of 460 questionnaires were sent out. 19 questionnaires were not answered seriously and 441 valid data were obtained, with an effective rate of $95.9 \%$. The BP neural network model was established by Matlab2018A to verify the algorithm.

\section{1 normalization}

Before the training of the model, the input samples were normalized to the interval $[-1,1]$

$$
\hat{Z}_{i}=\frac{2\left(Z i-Z_{\min }\right)}{Z_{\max }-Z_{\min }}-1
$$

$Z_{i}$ and $\hat{Z}_{i}$ represent the actual value and the normalized value of the ith data respectively, $z_{\min }$ and $\mathrm{Z}_{\max }$ are the maximum and minimum value in the data.

\subsection{Parameter Setting}

In the process of network training, the initial error is relatively large, so the condition for the model to set the end of sample data learning is that the error accuracy is less than 0.00001 , and the initial value of learning rate is.

\subsection{Fitting image}

4.3.1. BP forecast output. For 441 sets of statistical data, the predicted output and expected output were carried out, as shown in Figure 2, to judge the error.

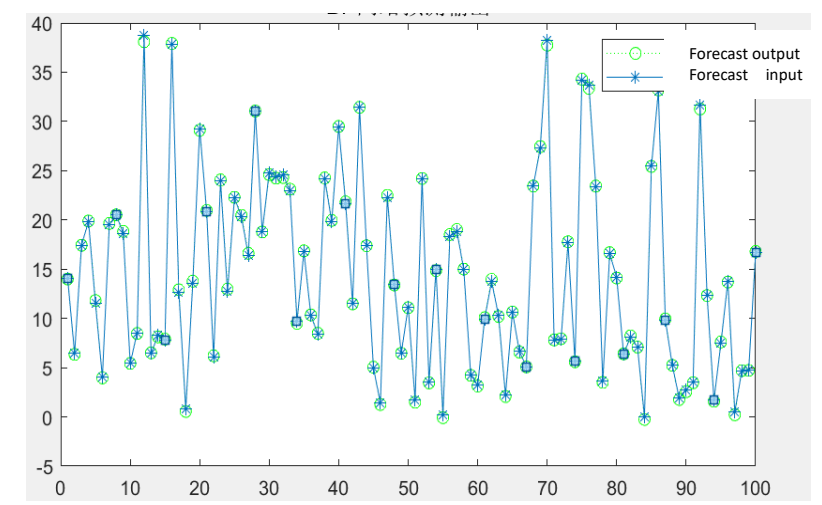

Figure 2 Prediction output of BP network

4.3.2 Percentage of prediction error. The error calculation of the predicted results is about $4.1 \%$, as shown in Figure 3, which can verify the high accuracy of the model. 


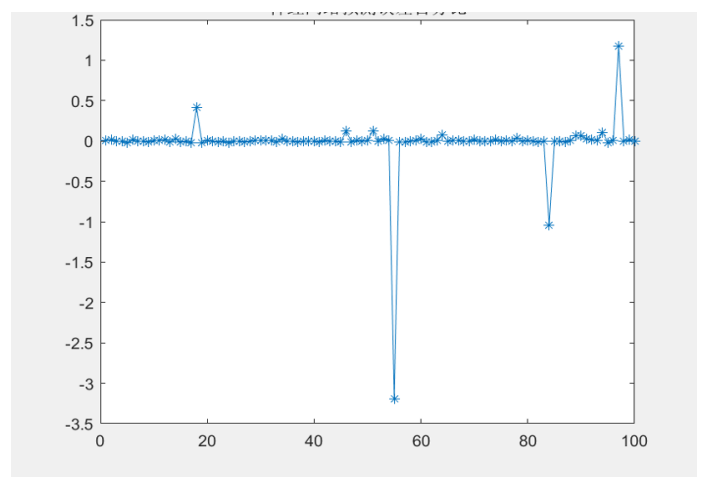

Figure 3 prediction error percentage of neural network

4.3.3 Training results. Twelve input factors were selected and the training results of 441 sets of data based on BP neural network were shown in Figure 4. Therefore, the underwriting system under this model has high accuracy.

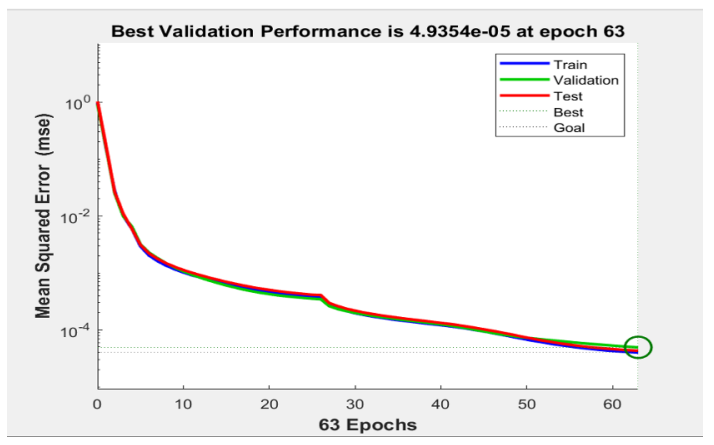

Figure 4 Training results

\section{Conclusion}

Based on literature reading, this paper summarized the factors affecting entrepreneurship into three types of identification indicators: entrepreneurial team, entrepreneurial project and entrepreneurial environment. Then, the Timmons evaluation index was summarized and classified, and 12 input variables were obtained by combining the existing research results. Again through the analysis of the algorithm, the design is based on the BP neural network of college students' entrepreneurship underwriting system, through times of training set the ideal error precision and initial values, learning rate of university graduates in the process of entrepreneurial success probability and entrepreneurial risk forecast accurately, and tests this with the survey of 441 data, found that has high accuracy.

\section{References}

1. Shane S., Venkataraman S. The Promise of Entrepreneurship as A Field of Research [J]. Academy of Management Re-view, 2000,25 (1): 217-226.

2. Ge Baoshan, Gao Yang, Jiang Dake. Timmons' Thought Evolution and Contribution: A Rethinking of Entrepreneurship [J]. Studies in Science of Science,2013,31(08):1207-1215.

3. Jiang Yanfu, Qiu Qiong. An empirical study on the sequence of important indicators of entrepreneurial opportunity evaluation $[\mathrm{J}]$. Studies in Science of Science, 2004(01):59-63.

4. Peng Yufang. The Application of Fuzzy Comprehensive Evaluation Method in Entrepreneurial Opportunity Evaluation [J]. Mathematics in Practice and Theory, 2011, 41(18): 31-35.

5. Nan Guojun, Hou Zejun, Liu Kai. Journal of Hefei University of Technology (Natural Science Edition),2020,43(12):1713-1717. (in Chinese)

6. Li Shuzhou, He Gang. Comprehensive evaluation of college students' innovation and entrepreneurship ability based on ANP-entropy weight method [J]. Journal of Anhui University of Science and Technology (Social Science Edition), 2020, 22(01): 42-46.

7. Bhave Mahesh P. A Process Model for Entrepreneurial Venture Creation [J]. Elsevier, 1994, 9(3).

8. Yan Xiaohan. A Study on the Influencing Factors of College Students' Entrepreneurship Intention from the Multi-dimensional Social Ecological Perspective [D]. Hefei University of Technology,2020.

9. Miao, Q. Research on the mechanism of entrepreneurial opportunity identification and decision making based on rule focus [D]. Zhejiang University,2006. 